\title{
Commutators of Bilinear Hardy Operators on Two Weighted Herz Spaces with Variable Exponents
}

\author{
Shengrong Wang and Jingshi $\mathrm{Xu}^{*}$ \\ Guangxi Colleges and Universities, Key Laboratory of Data Analysis and \\ Computation, School of Mathematics and Computing Science, Guilin \\ University of Electronic Technology, Guilin, Jiangxi 541004, China
}

Received 28 April 2020; Accepted (in revised version) 21 July 2021

Dedicated to Prof. Shanzhen Lu with admiration on the occasion of his 80th birthday

\begin{abstract}
In this paper, we obtain the boundedness of bilinear commutators generated by the bilinear Hardy operator and BMO functions on products of two weighted Herz spaces.
\end{abstract}

Key Words: Hardy operator, commutator, Muckenhoupt, BMO, variable exponent, Herz space.

AMS Subject Classifications: 42B35

\section{Introduction}

Denote by $L_{\text {loc }}^{1}\left(\mathbb{R}^{n}\right)$ the set of all complex-valued locally integrable functions on $\mathbb{R}^{n}$. The $n$ dimensional Hardy operator was introduced by Faris in [28] as

$$
H f(x):=\frac{1}{\Omega_{n}|x|^{n}} \int_{|y|<|x|} f(y) d y, \quad x \in \mathbb{R}^{n} \backslash\{0\} \quad \text { for each } f \in L_{\mathrm{loc}}^{1}\left(\mathbb{R}^{n}\right),
$$

where and what follows $\Omega_{n}$ is the volume of the unit ball in $\mathbb{R}^{n}$. When $n=1$, the Hardy operator was firstly considered in [10]. In [18], Christ and Grafakos showed that the Hardy operator is bounded on $L^{p}\left(\mathbb{R}^{n}\right)$ for $p \in(1, \infty)$. For the boundedness of $H$ on other functions, we refer the reader to the survey [25] by Shanzhen Lu.

For $m \in \mathbb{N}$, suppose $f_{1}, \cdots, f_{m}$ in $L_{\text {loc }}^{1}\left(\mathbb{R}^{n}\right)$, the $m$-linear Hardy operator is defined by

$$
H\left(f_{1}, \cdots, f_{m}\right):=\frac{1}{\Omega_{m n}|x|^{m n}} \int_{\left|\left(y_{1}, \cdots, y_{m}\right)\right|<|x|} \prod_{i=1}^{m} f_{i}\left(y_{i}\right) d y_{1} \cdots d y_{m}, \quad x \in \mathbb{R}^{n} \backslash\{0\} .
$$

${ }^{*}$ Corresponding author. Email addresses: jingshixu@126.com (J. Xu), 67775874@qq.com (S. Wang) 
If $b \in L_{\text {loc }}^{1}\left(\mathbb{R}^{n}\right)$, set

$$
\|b\|_{*}:=\sup _{B} \frac{1}{|B|} \int_{B}\left|b(x)-b_{B}\right| d x,
$$

where the supremum is taken over all balls in $\mathbb{R}^{n}, b_{B}$ is the mean of $b$ on $B$, and what follows $|E|$ is the Lebesgue measure of measurable set $E$ in $\mathbb{R}^{n}$. A function $b$ is called bounded mean oscillation if $\|b\|_{*}<\infty$. Denote by $\operatorname{BMO}\left(\mathbb{R}^{n}\right)$ the set of all bounded mean oscillation functions on $\mathbb{R}^{n}$.

The commutator of $m$-linear Hardy operator is defined by

$$
H_{\vec{b}}\left(f_{1}, \cdots, f_{m}\right)(x):=\sum_{i=1}^{m} H_{b_{i}}^{i}\left(f_{1}, \cdots, f_{m}\right)(x),
$$

where

$$
H_{b_{i}}^{i}\left(f_{1}, \cdots, f_{m}\right)(x):=b_{i}(x) H\left(f_{1}, \cdots, f_{m}\right)(x)-H\left(f_{1}, \cdots, f_{i-1}, f_{i} b_{i}, f_{i+1}, \cdots, f_{m}\right)(x) .
$$

When $m=1$, the operator $H_{b}(f)(x)=b(x) H f(x)-H(b f)(x)$. Although, our results hold for each $m \geq 2$, for brevity, we only consider $m=2$.

$\mathrm{Fu}$, Liu and $\mathrm{Lu}$ [31] studied the boundedness of the commutators of weighted Hardy operators (with symbols in $\mathrm{BMO}\left(\mathbb{R}^{n}\right)$ ) on $L^{p}\left(\mathbb{R}^{n}\right)$, where $1<p<\infty$. Fu, Gong and $\mathrm{Lu}$, ect. [34] proved the boundedness of the commutators of weighted multilinear Hardy operators (with symbols in central BMO space) on the product of central Morrey spaces $\dot{B}^{p, \lambda}$. Shi and Lu [27] introduced some characterizations of $\dot{\mathcal{C}}^{p, \lambda}$ for $-1 / p<\lambda<0$, via the boundedness of commutator operators of Hardy type. Zhao and Lu [7] precisely evaluate the operator norm of the fractional Hardy operator $\mathbb{H}^{\beta}$ from $L^{p}\left(\mathbb{R}^{n}\right)$ to $L^{q}\left(\mathbb{R}^{n}\right)$, where $0<\beta<n, 1<p<q<\infty$ and $1 / p-1 / q=\beta / n$. Zhao, $\mathrm{Fu}$ and $\mathrm{Lu}$ [8] gived some new properties of $M_{p}$ weight functions on $\mathbb{R}^{n}$ and used them to characterize the boundedness of bilinear Hardy inequalities on the weighted Lebesgue spaces. Yu and Lu [29] studied the $H^{1}$-boundedness of the generalized commutators of Hardy operator with a homogeneous kernel $\mathcal{H}_{\Omega, A, \beta}^{m}$. Lu, Yan and Zhao [26] explicitly worked out the bounds of the operator $\mathbb{H}$ from $L^{p}$ to $L^{q}$ and from $L^{1}$ to $L^{\frac{n}{n-\beta}, \infty}$. Fu, Wu and Lu proved the boundedness of commutators generated by the $p$-adic Hardy operators (Hardy-Littlewood-Pólya operators) and the central BMO functions on $L^{q}\left(|x|_{p}^{\alpha} d x\right)$ and Herz spaces $K_{r}^{\alpha, q}$. Zhao, Fu and $\mathrm{Lu}$ [9] proved that the higher dimensional Hardy operator is bounded from Hardy spaces to Lebesgue spaces, and discussed the endpoint estimate for the commutators generated by the Hardy operator and (central) BMO functions. Fu, Grafakos and Lu, etc. [32] obtained norms of $m$-linear Hardy operators and $m$-linear Hilbert operators on Lebesgue spaces with power weights.

In [19], Izuki proved the boundedness of commutators generated by singular integrals and BMO functions on Herz spaces $\dot{K}_{p(\cdot)}^{\alpha, q}\left(\mathbb{R}^{n}\right)$ with variable exponent $p(\cdot)$. Shu, Wang and Meng [17] obtained the boundedness of commutators of Hardy type operators 
and BMO functions on Herz spaces $\dot{K}_{p(\cdot), q}^{\alpha(\cdot)}$ with variable exponents $\alpha(\cdot)$ and $p(\cdot)$. Izuki and Noi [24] proved the boundedness of fractional integral operators on weighted Herz spaces $\dot{K}_{p(\cdot)}^{\alpha, q}(w)$ with variable exponent. Almeida and Drihem [1] obtained the boundedness of sublinear operator on variable Herz spaces $K_{p(\cdot)}^{\alpha(\cdot), q}(w)$.

In 2020, Izuki and Noi introduced two weighted Herz spaces with variable exponents in [23]. Motivated by the mentioned works, we will consider the boundedness of commutators generated by bilinear Hardy operator and BMO functions on two weighted Herz spaces with variable exponents. The plan of the paper is as follows. In Section 2, we collect some notations and state the main result. The proof of the main result will be given in Section 3.

\section{Notations and main result}

In this section, we firstly recall some definitions and notations, then we state our result. Let $p(\cdot)$ be a measurable function on $\mathbb{R}^{n}$ and take values in $[1, \infty)$. The variable Lebesgue space $L^{p(\cdot)}\left(\mathbb{R}^{n}\right)$ is defined by

$$
L^{p(\cdot)}\left(\mathbb{R}^{n}\right):=\left\{f \text { is measurable: } \int_{\mathbb{R}^{n}}\left(\frac{|f(x)|}{\lambda}\right)^{p(x)} d x<\infty \text { for some } \lambda>0\right\} .
$$

The space $L^{p(\cdot)}\left(\mathbb{R}^{n}\right)$ becomes a Banach function space equipped with the norm

$$
\|f\|_{L^{p(\cdot)}}:=\inf \left\{\lambda>0: \int_{\Omega}\left(\frac{|f(x)|}{\lambda}\right)^{p(x)} d x \leq 1\right\}
$$

The space $L_{\text {loc }}^{p(\cdot)}\left(\mathbb{R}^{n}\right)$ is defined by

$$
L_{\text {loc }}^{p(\cdot)}\left(\mathbb{R}^{n}\right):=\left\{f: f_{\chi_{K}} \in L^{p(\cdot)}\left(\mathbb{R}^{n}\right) \text { for all compact subsets } K \subset \mathbb{R}^{n}\right\},
$$

where and what follows, $\chi_{S}$ denotes the characteristic function of a measurable set $S \subset$ $\mathbb{R}^{n}$. Let $p(\cdot): \mathbb{R}^{n} \rightarrow(0, \infty)$, we denote

$$
p_{-}:=\operatorname{ess} \inf _{x \in \mathbb{R}^{n}} p(x), \quad p_{+}:=\text {ess } \sup _{x \in \mathbb{R}^{n}} p(x) .
$$

The set $\mathcal{P}\left(\mathbb{R}^{n}\right)$ consists of all $p(\cdot)$ satisfying $p_{-}>1$ and $p_{+}<\infty ; \mathcal{P}^{0}\left(\mathbb{R}^{n}\right)$ consists of all $p(\cdot)$ satisfying $p_{-}>0$ and $p_{+}<\infty$. $L^{p(\cdot)}$ can be similarly defined as above for $p(\cdot) \in \mathcal{P}^{0}\left(\mathbb{R}^{n}\right) \cdot p^{\prime}(\cdot)$ is the conjugate exponent of $p(\cdot)$, that means $1 / p(\cdot)+1 / p^{\prime}(\cdot)=1$.

Let $f \in L_{\text {loc }}^{1}\left(\mathbb{R}^{n}\right)$. Then the standard Hardy-Littlewood maximal function of $f$ is defined by

$$
M f(x):=\sup _{B \ni x} \frac{1}{|B|} \int_{B}|f(y)| d y, \quad \forall x \in \mathbb{R}^{n},
$$


where $B$ is a ball. In general, the Hardy-littlewood maximal operator $M$ is not bounded on variable Lebesgue spaces. But if $p(\cdot) \in \mathcal{P}\left(\mathbb{R}^{n}\right)$ and satisfies the following global logHölder continuous, then $M$ is bounded on $L^{p(\cdot)}\left(\mathbb{R}^{n}\right)$, see [6]. For more details see $[2,14-$ 16].

Definition 2.1. Let $\alpha(\cdot)$ be a real-valued measurable function on $\mathbb{R}^{n}$.

(i) The function $\alpha(\cdot)$ is locally log-Hölder continuous if there exists a constant $C_{1}$ such that

$$
|\alpha(x)-\alpha(y)| \leq \frac{C_{1}}{\log (e+1 /|x-y|)}, \quad x, y \in \mathbb{R}^{n}, \quad|x-y|<\frac{1}{2} .
$$

(ii) The function $\alpha(\cdot)$ is log-Hölder continuous at the origin if

$$
|\alpha(x)-\alpha(0)| \leq \frac{C_{2}}{\log (e+1 /|x|)}, \quad \forall x \in \mathbb{R}^{n} .
$$

Denote by $\mathcal{P}_{0}^{\log }\left(\mathbb{R}^{n}\right)$ the set of all log-Hölder continuous functions at the origin.

(iii) The function $\alpha(\cdot)$ is $\log$-Hölder continuous at infinity if there exists a constant $C_{3}$ such that

$$
\left|\alpha(x)-\alpha_{\infty}\right| \leq \frac{C_{3}}{\log (e+|x|)}, \quad \forall x \in \mathbb{R}^{n} .
$$

Denote by $\mathcal{P}_{\infty}^{\log }\left(\mathbb{R}^{n}\right)$ the set of all log-Hölder continuous functions at infinity.

(iv) The function $\alpha(\cdot)$ is global log-Hölder continuous if $\alpha(\cdot)$ are both locally log-Hölder continuous and $\log$-Hölder continuous at infinity. Denote by $\mathcal{P}^{\log }\left(\mathbb{R}^{n}\right)$ the set of all global log-Hölder continuous functions.

Definition 2.2. Fix $p \in(1, \infty)$. A positive measurable function $w$ is said to be in the Muckenhoupt class $A_{p}$, if there exists a positive constant $C$ for all balls $B$ in $\mathbb{R}^{n}$ such that

$$
\left(\frac{1}{B} \int_{B} w(x) d x\right)\left(\frac{1}{B} \int_{B} w(x)^{1-p^{\prime}} d x\right)^{p-1} \leq C .
$$

We say $w \in A_{1}$, if $M w(x) \leq C w(x)$ for a.e. $x$. If $1 \leq p<q<\infty$, then $A_{p} \subset A_{q}$. We denote $A_{\infty}=\cup_{p>1} A_{p}$. The Muckenhoupt $A_{p}$ class with constant exponent $p \in(1, \infty)$ was firstly proposed by Muckenhoupt in [5].

Definition 2.3. Let $p(\cdot) \in \mathcal{P}\left(\mathbb{R}^{n}\right)$, a positive measurable function $w$ is said to be in $A_{p(\cdot)}$, if there exists a positive constant $C$ for all balls $B$ in $\mathbb{R}^{n}$ such that

$$
\frac{1}{|B|}\left\|w^{1 / p(\cdot)} \chi_{B}\right\|_{L^{p(\cdot)}}\left\|w^{-1 / p(\cdot)} \chi_{B}\right\|_{L^{p^{\prime}(\cdot)}} \leq C .
$$


Definition 2.4. Let $p \in \mathcal{P}\left(\mathbb{R}^{n}\right)$ and $w$ be a weight. The weight variable Lebesgue space $L^{p(\cdot)}(w)$ is defined by

$$
L^{p(\cdot)}(w):=\left\{\text { fis measurable on } \mathbb{R}^{n} \text { and }\|f\|_{L^{p(\cdot)}(w)}:=\left\|f w^{1 / p(\cdot)}\right\|_{L^{p(\cdot)}\left(\mathbb{R}^{n}\right)}<\infty\right\} .
$$

Then $L^{p(\cdot)}(w)$ is a Banach space.

To give the definitions of the weighted Herz space with variable exponents, we use the following notations. For each $k \in \mathbb{Z}$ we define $B_{k}:=\left\{x \in \mathbb{R}^{n}:|x| \leq 2^{k}\right\}, D_{k}:=$ $B_{k} \backslash B_{k-1}=\left\{x \in \mathbb{R}^{n}: 2^{k-1}<|x| \leq 2^{k}\right\}, \chi_{k}:=\chi_{D_{k}}, \widetilde{\chi}_{m}=\chi_{m}, m \geq 1, \widetilde{\chi}_{0}=\chi_{B_{0}}$. We also need the notation of the variable mixed sequence space $\ell^{q(\cdot)}\left(L^{p(\cdot)}(w)\right)$. Let $w$ be a positive measurable function. Given a sequence of functions $\left\{f_{j}\right\}_{j \in \mathbb{Z}}$, define the modular

$$
\rho_{\ell^{q}(\cdot)\left(L^{p(\cdot)}(w)\right)}\left(\left(f_{j}\right)_{j}\right):=\sum_{j \in \mathbb{Z}} \inf \left\{\lambda_{j}: \int_{\mathbb{R}^{n}}\left(\frac{\left|f_{j}(x)\right|}{\lambda_{j}^{\frac{1}{q(x)}}}\right)^{p(x)} w(x) d x \leq 1\right\},
$$

where $\lambda^{1 / \infty}=1$. If $q^{+}<\infty$ or $q(\cdot) \leq p(\cdot)$, the above can be written as

$$
\rho_{\ell q(\cdot)\left(L^{p(\cdot)}(w)\right)}\left(\left(f_{j}\right)_{j}\right)=\sum_{j \in \mathbb{Z}}\left\|\left|f_{j} w^{1 / p(\cdot)}\right|^{q(\cdot)}\right\|_{L^{\frac{p(\cdot)}{q(\cdot)}}} .
$$

The norm is

$$
\left\|\left(f_{j}\right)_{j}\right\|_{\ell q(\cdot)\left(L^{p(\cdot)}(w)\right)}:=\inf \left\{\mu>0: \rho_{\ell q(\cdot)\left(L^{p(\cdot)}(w)\right)}\left(\left(f_{j} / \mu\right)_{j}\right) \leq 1\right\} .
$$

Definition 2.5. Let $w_{1}$, w $w_{2}$ be weights on $\mathbb{R}^{n}, p(\cdot) \in \mathcal{P}\left(\mathbb{R}^{n}\right), q(\cdot) \in \mathcal{P}_{0}\left(\mathbb{R}^{n}\right)$. Let $\alpha(\cdot)$ be a bounded real-valued measurable function on $\mathbb{R}^{n}$. The homogeneous two weighted variable Herz space $\dot{K}_{p(\cdot), \lambda}^{\alpha(\cdot), q(\cdot)}\left(w_{1}, w_{2}\right)$ and non-homogeneous two weighted variable Herz space $K_{p(\cdot), \lambda}^{\alpha(\cdot),(\cdot)}\left(w_{1}, w_{2}\right)$ are defined, respectively, by

$$
\begin{aligned}
& \dot{K}_{p(\cdot)}^{\alpha(\cdot), q(\cdot)}\left(w_{1}, w_{2}\right):=\left\{f \in L_{\mathrm{loc}}^{p(\cdot)}\left(\mathbb{R}^{n} \backslash\{0\}, w_{2}\right):\|f\|_{\dot{K}_{p(\cdot)}^{\alpha(\cdot), q(\cdot)}\left(w_{1}, w_{2}\right)}<\infty\right\}, \\
& K_{p(\cdot)}^{\alpha(\cdot), q(\cdot)}\left(w_{1}, w_{2}\right):=\left\{f \in L_{\mathrm{loc}}^{p(\cdot)}\left(\mathbb{R}^{n}, w_{2}\right):\|f\|_{K_{p(\cdot)}^{\alpha(\cdot), q(\cdot)}\left(w_{1}, w_{2}\right)}<\infty\right\},
\end{aligned}
$$

where

$$
\begin{aligned}
& \|f\|_{\dot{K}_{p(\cdot)}^{\alpha(\cdot),(\cdot)}\left(w_{1}, w_{2}\right)}:=\left\|\left\{w_{1}\left(B_{k}\right)^{\alpha(\cdot) / n} f \chi_{k}\right\}_{k \in \mathbb{Z}}\right\|_{\ell q(\cdot)\left(L^{p(\cdot)}\left(w_{2}\right)\right)}, \\
& \|f\|_{K_{p(\cdot)}^{\alpha(\cdot), q(\cdot)}\left(w_{1}, w_{2}\right)}:=\left\|\left\{w_{1}\left(B_{k}\right)^{\alpha(\cdot) / n} f \widetilde{\chi}_{k}\right\}_{k \geq 0}\right\|_{\ell q(\cdot)\left(L^{p(\cdot)}\left(w_{2}\right)\right)} .
\end{aligned}
$$

For any quantities $A$ and $B$, if there exists a constant $C>0$ such that $A \leq C B$, we write $A \lesssim B$. If $A \lesssim B$ and $B \lesssim A$, we write $A \approx B$. 
Lemma 2.1 ([23, Theorem 3]). Let $\alpha(\cdot) \in L^{\infty}\left(\mathbb{R}^{n}\right), p(\cdot), q(\cdot) \in \mathcal{P}_{0}\left(\mathbb{R}^{n}\right), w_{1} \in A_{r}$ for some $r \in[1, \infty)$ and $w_{2}$ be a weight. If $\alpha(\cdot)$ and $q(\cdot)$ are log-Hölder continuous at infinity, then

$$
K_{p(\cdot)}^{\alpha(\cdot), q(\cdot)}\left(w_{1}, w_{2}\right)=K_{p(\cdot)}^{\alpha_{\infty}, q_{\infty}}\left(w_{1}, w_{2}\right) .
$$

Additionally, if $\alpha(\cdot)$ and $q(\cdot)$ are log-Hölder continuous at the origin, then

$$
\begin{aligned}
\|f\|_{\dot{K}_{p(\cdot)}^{\alpha(\cdot), q(\cdot)}\left(w_{1}, w_{2}\right)} \approx( & \left.\sum_{k \leq 0}\left\|w_{1}\left(B_{k}\right)^{\alpha(0) / n} f \chi_{k}\right\|_{L^{p(\cdot)}\left(w_{2}\right)}^{q(0)}\right)^{\frac{1}{q(0)}} \\
& +\left(\sum_{k>0}\left\|w_{1}\left(B_{k}\right)^{\alpha_{\infty} / n} f \chi_{k}\right\|_{L^{p(\cdot)}\left(w_{2}\right)}^{q_{\infty}}\right)^{\frac{1}{q_{\infty}}} .
\end{aligned}
$$

The following Lemma 2.2 comes from the result of $[3,11,12]$.

Lemma 2.2. If $q \in[1, \infty)$ and $w \in A_{q}$, then there exist constants $\delta \in(0,1)$ and $C>0$ such that for all balls $B$ in $\mathbb{R}^{n}$ and all measurable subsets $S \subset B$,

$$
\begin{aligned}
& \frac{w(B)}{w(S)} \leq C\left(\frac{B}{S}\right)^{q}, \\
& \frac{w(S)}{w(B)} \leq C\left(\frac{S}{B}\right)^{\delta} .
\end{aligned}
$$

Lemma 2.3 ([23, (21) and (22)]). If $p(\cdot) \in \mathcal{P}^{\log }\left(\mathbb{R}^{n}\right) \cap \mathcal{P}\left(\mathbb{R}^{n}\right)$ and $w \in A_{p(\cdot)}$, then there exist constants $\delta_{1}, \delta_{2} \in(0,1)$ and $C>0$ such that for all $k, l \in \mathbb{Z}$ with $k \leq l$,

$$
\begin{aligned}
& \frac{\left\|\chi_{k}\right\|_{L^{p(\cdot)}\left(w^{p(\cdot)}\right)}}{\left\|\chi_{l}\right\|_{L^{p(\cdot)}\left(w^{p(\cdot)}\right)}} \leq C\left(\frac{\left|D_{k}\right|}{\left|D_{l}\right|}\right)^{\delta_{1}}, \\
& \frac{\left\|\chi_{k}\right\|_{\left(L^{p(\cdot)}\left(w^{p(\cdot)}\right)\right)^{\prime}}}{\left\|\chi_{l}\right\|_{\left(L^{p(\cdot)}\left(w^{p(\cdot)}\right)\right)^{\prime}}} \leq C\left(\frac{\left|D_{k}\right|}{\left|D_{l}\right|}\right)^{\delta_{2}} .
\end{aligned}
$$

Remark 2.1. If $w \in A_{p(\cdot)}$, then $w^{-p^{\prime}(\cdot) / p(\cdot)} \in A_{p^{\prime}(\cdot)}$. Therefore, the Hardy-littlewood maximal operator $M$ is bounded on $L^{p^{\prime}(\cdot)}\left(w^{-p^{\prime}(\cdot) / p(\cdot)}\right)$.

Our main result is as follows.

Theorem 2.1. Suppose that $H$ is the bilinear Hardy operator, $p_{1}(\cdot), p_{2}(\cdot) \in \mathcal{P}^{\log }\left(\mathbb{R}^{n}\right) \cap$ $\mathcal{P}\left(\mathbb{R}^{n}\right)$. Let $v \in A_{r}$ for some $r \in[1, \infty), w_{i} \in A_{p_{i}(\cdot)}$ and $w=\prod_{i=1}^{2} w_{i}^{p(\cdot) / p_{i}(\cdot)}$. Assume that $\alpha(\cdot) \in L^{\infty}\left(\mathbb{R}^{n}\right) \cap \mathcal{P}_{0}^{\log }\left(\mathbb{R}^{n}\right) \cap \mathcal{P}_{\infty}^{\log }\left(\mathbb{R}^{n}\right), \alpha(\cdot)=\alpha_{1}(\cdot)+\alpha_{2}(\cdot), q(\cdot) \in \mathcal{P}_{0}^{\log }\left(\mathbb{R}^{n}\right) \cap \mathcal{P}_{\infty}^{\log }\left(\mathbb{R}^{n}\right)$, $1 / q(0)=1 / q_{1}(0)+1 / q_{2}(0), 1 / q_{\infty}=1 / q_{1 \infty}+1 / q_{2 \infty}, \delta_{i 1}, \delta_{i 2} \in(0,1)$ are the constants in Lemma 2.3 for exponents $p_{i}(\cdot)$ and weights $w_{i}, i=1,2$. If $w^{+} \alpha^{+}<n \delta_{i 2}$ for $i=1,2$, $1 / p(\cdot)=1 / p_{1}(\cdot)+1 / p_{2}(\cdot)$, then $H_{\vec{b}}^{1}$ is bounded from $\dot{K}_{p_{1}(\cdot)}^{\alpha_{1}(\cdot), q_{1}(\cdot)}\left(v, w_{1}\right) \times \dot{K}_{p_{2}(\cdot)}^{\alpha_{2}(\cdot), q_{2}(\cdot)}\left(v, w_{2}\right)$ into $\dot{K}_{p(\cdot)}^{\alpha(\cdot), q(\cdot)}(v, w)$, where $\vec{b}=\left(b_{1}, b_{2}\right), b_{1}, b_{2} \in \operatorname{BMO}\left(\mathbb{R}^{\mathrm{n}}\right)$. 


\section{Proof of Theorem 2.1}

To prove Theorem 2.1, we need a series of Lemmas.

Lemma 3.1 ([23, Lemma 7]). Let $k, l \in \mathbb{Z}, w \in A_{q}$ with $q \in[1, \infty), \delta \in(0,1)$ be the constants in Lemma 2.2,

$$
w^{-}=\left\{\begin{array}{ll}
\delta, & \text { if } \alpha^{-} \geq 0, \\
q, & \text { if } \alpha^{-}<0,
\end{array} \quad w^{+}= \begin{cases}q, & \text { if } \alpha^{+} \geq 0, \\
\delta, & \text { if } \alpha^{+}<0 .\end{cases}\right.
$$

If $\alpha(\cdot) \in L^{\infty}\left(\mathbb{R}^{n}\right)$ is log-Hölder continuous both at the origin and infinity, then for any $x \in D_{k}$ and $y \in D_{l}$,

$$
w\left(B_{k}\right)^{\alpha(x)} \leq C w\left(B_{l}\right)^{\alpha(y)} \times \begin{cases}2^{(k-l) n w^{+} \alpha^{+}}, & \text {if } l \leq k-1, \\ 1, & \text { if } k-1<l \leq k+1, \\ 2^{(k-l) n w^{-} \alpha^{-}}, & \text {if } l>k+1 .\end{cases}
$$

Lemma 3.2 ([23, Lemma 8]). Let $w \in A_{q}$ with $q \in[1, \infty)$. If $\alpha(\cdot) \in L^{\infty}\left(\mathbb{R}^{n}\right)$ is log-Hölder continuous both at the origin and infinity. then for all $k \in \mathbb{Z}$ and $x \in D_{k}$,

$$
\begin{aligned}
& w\left(D_{k}\right)^{\alpha(x)} \approx w\left(D_{k}\right)^{\alpha_{\infty}}, \quad \text { if } k \geq 0, \\
& w\left(D_{k}\right)^{\alpha(x)} \approx w\left(D_{k}\right)^{\alpha(0)}, \quad \text { if } k \leq-1 .
\end{aligned}
$$

Lemma 3.3 below have been proved by Izuki in $[21,22]$

Lemma 3.3. Let $X$ be a Banach function space on $\mathbb{R}^{n}$. If the Hardy-littlewood maximal operator $M$ is weakly bounded on $X$, that is,

$$
\left\|\chi_{\{M f>\lambda\}}\right\|_{X} \lesssim \lambda^{-1}\|f\|_{X}
$$

holds for all $f \in X$ and $\lambda>0$. Then we have

$$
\sup _{\text {ball } B} \frac{1}{|B|}\left\|\chi_{B}\right\|_{X}\left\|\chi_{B}\right\|_{X^{\prime}}<\infty,
$$

where $X^{\prime}$ denotes the associated space of $X$.

Ho [13] has initially proved the following characterization of the BMO norm via Banach function spaces. Izuki [20] gave another simple proof.

Lemma 3.4. Let $X$ be a Banach function space on $\mathbb{R}^{n}$. If $M$ is bounded on the associate sapce $X^{\prime}$, then for all $b \in \mathrm{BMO}\left(\mathbb{R}^{n}\right)$,

$$
\|b\|_{*} \approx \sup _{\text {ball } B \subset \mathbb{R}^{n}} \frac{1}{\left\|\chi_{B}\right\|_{X}}\left\|\left(b-b_{B}\right) \chi_{B}\right\|_{X}
$$


Lemma 3.5 (The generalized Hölder inequality). Let $X$ be a Banach function space on $\mathbb{R}^{n}$. If $f \in X$ and $g \in X^{\prime}$, then we have

$$
\int_{\mathbb{R}^{n}}|f(x) g(x)| d x \leq\|f\|_{X}\|g\|_{X^{\prime}}
$$

where $X^{\prime}$ denotes the associated space of $X$.

Lemma 3.6 ([4, Theorem 2.3]). Let $p(\cdot), p_{1}(\cdot), p_{2}(\cdot) \in \mathcal{P}_{0}\left(\mathbb{R}^{n}\right)$ such that $1 / p(x)=1 / p_{1}(x)+$ $1 / p_{2}(x)$ for $x \in \mathbb{R}^{n}$. Then there exists a constant $C_{p, p_{1}}$ independent of functions $f$ and $g$ such that

$$
\|f g\|_{L^{p(\cdot)}} \leq C_{p, p_{1}}\|f\|_{L^{p_{1}(\cdot)}}\|g\|_{L^{p_{2}(\cdot)}}
$$

holds for every $f \in L^{p_{1}(\cdot)}\left(\mathbb{R}^{n}\right)$ and $g \in L^{p_{2}(\cdot)}\left(\mathbb{R}^{n}\right)$.

If $p(\cdot), p_{1}(\cdot), p_{2}(\cdot) \in \mathcal{P}\left(\mathbb{R}^{n}\right)$ such that $1 / p(x)=1 / p_{1}(x)+1 / p_{2}(x)$ for $x \in \mathbb{R}^{n}$, and $w \in A_{p(\cdot)}$ with $w_{i} \in A_{p_{i}(\cdot)}, w=\prod_{i=1}^{2} w_{i}^{p(\cdot) / p_{i}(\cdot)}$, then

$$
\|f g\|_{L^{p(\cdot)}(w)} \leq C_{p, p_{1}}\|f\|_{L^{p_{1}(\cdot)}\left(w_{1}\right)}\|g\|_{L^{p_{2}(\cdot)}\left(w_{2}\right)} \cdot
$$

Lemma 3.7 ([30, Proposition 1.2]). Let $0<p \leq \infty, \delta>0$. Then there is a positive constant $C$ such that

$$
\left(\sum_{j=-\infty}^{\infty}\left(\sum_{k=-\infty}^{\infty} 2^{-|k-j| \delta} a_{k}\right)^{p}\right)^{1 / p} \leq C\left(\sum_{j=-\infty}^{\infty} a_{j}^{p}\right)^{1 / p}
$$

for non-negative sequences $\left\{a_{j}\right\}_{j=-\infty}^{\infty}$. Here, when $p=\infty$, it is understood that (3.1) stands for

$$
\sup _{j \in \mathbb{Z}}\left(\sum_{k=-\infty}^{\infty} 2^{-|k-j| \delta} a_{k}\right) \leq C \sup _{j \in \mathbb{Z}} a_{j} .
$$

Now we give the proof Theorem 2.1.

Proof of Theorem 2.1. Let $f_{1} \in \dot{K}_{p_{1}(\cdot)}^{\alpha_{1}(\cdot), q_{1}(\cdot)}\left(v, w_{1}\right), f_{2} \in M \dot{K}_{p_{2}(\cdot)}^{\alpha_{2}(\cdot), q_{2}(\cdot)}\left(v, w_{2}\right)$. Let $\left(b_{i}\right)_{B_{k}}$ express the average of $b_{i}$ on the ball $B_{k}$ for $i=1,2$, and $k \in \mathbb{Z}$. If $x \in D_{k}$, then

$$
\begin{aligned}
\left|H_{b_{1}}^{1}\left(f_{1}, f_{2}\right)(x)\right| & \lesssim 2^{-2 k n} \int_{\left|\left(y_{1}, y_{2}\right)\right|<|x|}\left|f_{1}\left(y_{1}\right) f_{2}\left(y_{2}\right)\right|\left|b_{1}(x)-b_{1}\left(y_{1}\right)\right| d y_{1} d y_{2} \\
& \lesssim 2^{-2 k n} \int_{\left|\left(y_{1}, y_{2}\right)\right|<|x|}\left|f_{1}\left(y_{1}\right) f_{2}\left(y_{2}\right)\right|\left|b_{1}(x)-\left(b_{1}\right)_{B_{k}}\right| d y_{1} d y_{2} \\
& +2^{-2 k n} \int_{\left|\left(y_{1}, y_{2}\right)\right|<|x|}\left|f_{1}\left(y_{1}\right) f_{2}\left(y_{2}\right)\right|\left|b_{1}\left(y_{1}\right)-\left(b_{1}\right)_{B_{k}}\right| d y_{1} d y_{2}
\end{aligned}
$$




$$
\begin{aligned}
& \lesssim 2^{-2 k n}\left|b_{1}(x)-\left(b_{1}\right)_{B_{k}}\right| \sum_{i=-\infty}^{k} \int_{D_{i}}\left|f_{1}\left(y_{1}\right)\right| d y_{1} \sum_{j=-\infty}^{k} \int_{D_{j}}\left|f_{2}\left(y_{2}\right)\right| d y_{2} \\
& \quad+2^{-2 k n} \sum_{i=-\infty}^{k} \int_{D_{i}}\left|f_{1}\left(y_{1}\right)\right|\left|b_{1}\left(y_{1}\right)-\left(b_{1}\right)_{B_{i}}\right| d y_{1} \sum_{j=-\infty}^{k} \int_{D_{j}}\left|f_{2}\left(y_{2}\right)\right| d y_{2} \\
& \quad+2^{-2 k n} \sum_{i=-\infty}^{k} \int_{D_{i}}\left|f_{1}\left(y_{1}\right)\right|\left|\left(b_{1}\right)_{B_{k}}-\left(b_{1}\right)_{B_{i}}\right| d y_{1} \sum_{j=-\infty}^{k} \int_{D_{j}}\left|f_{2}\left(y_{2}\right)\right| d y_{2} \\
& =: I_{1}+I_{2}+I_{3} .
\end{aligned}
$$

If $k>i, k>j$, then by Lemma 3.1, we have

$$
\begin{aligned}
& v\left(B_{k}\right)^{\alpha_{1}(x) / n} \int_{D_{i}}\left|f_{1}\left(y_{1}\right)\right| d y_{1} \lesssim 2^{(k-i) w^{+} \alpha^{+}} \int_{D_{i}} v\left(B_{i}\right)^{\alpha_{1}\left(y_{1}\right) / n}\left|f_{1}\left(y_{1}\right)\right| d y_{1}, \\
& v\left(B_{k}\right)^{\alpha_{2}(x) / n} \int_{D_{j}}\left|f_{2}\left(y_{2}\right)\right| d y_{2} \lesssim 2^{(k-j) w^{+} \alpha^{+}} \int_{D_{j}} v\left(B_{j}\right)^{\alpha_{2}\left(y_{2}\right) / n}\left|f_{2}\left(y_{2}\right)\right| d y_{2}, \\
& v\left(B_{k}\right)^{\alpha_{1}(x) / n} \int_{D_{i}}\left|f_{1}\left(y_{1}\right)\right|\left|G_{1}\right| d y_{1} \lesssim 2^{(k-i) w^{+} \alpha^{+}} \int_{D_{i}} v\left(B_{i}\right)^{\alpha_{1}\left(y_{1}\right) / n}\left|f_{1}\left(y_{1}\right)\right|\left|G_{1}\right| d y_{1}, \\
& v\left(B_{k}\right)^{\alpha_{1}(x) / n} \int_{D_{i}}\left|f_{1}\left(y_{1}\right)\right|\left|G_{2}\right| d y_{1} \lesssim 2^{(k-i) w^{+} \alpha^{+}} \int_{D_{i}} v\left(B_{i}\right)^{\alpha\left(y_{1}\right) / n}\left|f_{1}\left(y_{1}\right)\right|\left|G_{2}\right| d y_{1},
\end{aligned}
$$

where $G_{1}=b_{1}\left(y_{1}\right)-\left(b_{1}\right)_{B_{i}}$ and $G_{2}=\left(b_{1}\right)_{B_{k}}-\left(b_{1}\right)_{B_{i}}$.

Since

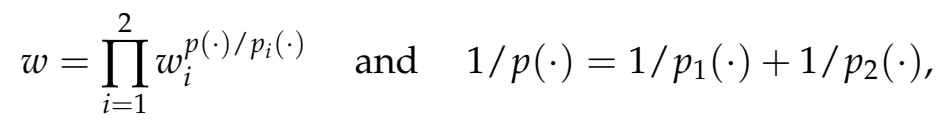

by Hölder's inequality, Lemmas 3.3 and 2.3, we have

$$
\begin{aligned}
& 2^{-2 k n}\left\|\chi_{i}\right\|_{L^{p_{1}^{\prime}(\cdot)}\left(w_{1}^{-p_{1}^{\prime}(\cdot) / p_{1}(\cdot)}\right)}\left\|\chi_{j}\right\|_{L^{p_{2}^{\prime}(\cdot)}\left(w_{2}^{-p_{2}^{\prime}(\cdot) / p_{2}(\cdot)}\right)}\left\|\chi_{k}\right\|_{L^{p(\cdot)}(w)} \\
& \lesssim 2^{-2 k n}\left\|\chi_{i}\right\|_{L_{1}^{p_{1}^{\prime}(\cdot)}\left(w_{1}^{-p_{1}^{\prime}(\cdot) / p_{1}(\cdot)}\right)}\left\|\chi_{j}\right\|_{L_{2}^{p^{\prime}(\cdot)}\left(w_{2}^{-p_{2}^{\prime}(\cdot) / p_{2}(\cdot)}\right)}\left\|\chi_{k}\right\|_{L^{p_{1}(\cdot)}\left(w_{1}\right)}\left\|\chi_{k}\right\|_{L^{p_{2}(\cdot)}\left(w_{2}\right)}
\end{aligned}
$$

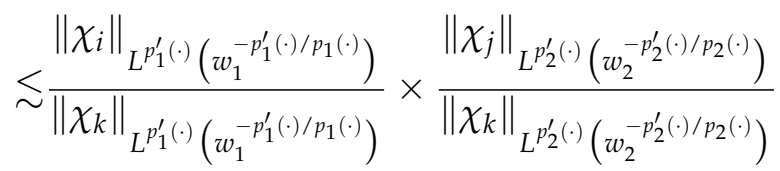

$$
\begin{aligned}
& \lesssim 2^{-(k-i) n \delta_{12}} \times 2^{-(k-j) n \delta_{22}} \text {. }
\end{aligned}
$$

Since

$$
w=\prod_{i=1}^{2} w_{i}^{p(\cdot) / p_{i}(\cdot)} \quad \text { and } \quad \alpha(\cdot)=\alpha_{1}(\cdot)+\alpha_{2}(\cdot),
$$


by (3.2a)-(3.2d) and Hölder's inequality, Lemma 3.4 and (3.3), we have

$$
\begin{aligned}
& \left\|v\left(B_{k}\right)^{\alpha(\cdot) / n} I_{1} \chi_{k}\right\|_{L^{p(\cdot)}(w)} \\
& \lesssim 2^{-2 k n}\left\|\left(b_{1}-\left(b_{1}\right)_{B_{k}}\right) \cdot \chi_{k}\right\|_{L^{p(\cdot)}(w)} \\
& \times \sum_{i=-\infty}^{k} 2^{(k-i) w^{+} \alpha^{+}}\left\|v\left(B_{i}\right)^{\alpha_{1}(\cdot) / n} f_{1} \chi_{i}\right\|_{L^{p_{1}(\cdot)}\left(w_{1}\right)}\left\|\chi_{i}\right\|_{L^{p_{1}^{\prime}(\cdot)}\left(w_{1}^{-p_{1}^{\prime}(\cdot) / p_{1}(\cdot)}\right)} \\
& \times \sum_{j=-\infty}^{k} 2^{(k-j) w^{+} \alpha^{+}}\left\|v\left(B_{j}\right)^{\alpha_{2}(\cdot) / n} f_{2} \chi_{j}\right\|_{L^{p_{2}(\cdot)}\left(w_{2}\right)}\left\|\chi_{j}\right\|_{L^{p_{2}^{\prime}(\cdot)}\left(w_{2}^{-p_{2}^{\prime}(\cdot) / p_{2}(\cdot)}\right)} \\
& \lesssim 2^{-2 k n}\left\|b_{1}\right\|_{*} \sum_{i=-\infty}^{k} 2^{(k-i) w^{+} \alpha^{+}}\left\|v\left(B_{i}\right)^{\alpha_{1}(\cdot) / n} f_{1} \chi_{i}\right\|_{L^{p_{1}(\cdot)}\left(w_{1}\right)}\left\|\chi_{i}\right\|_{L^{p_{1}^{\prime}(\cdot)}\left(w_{1}^{-p_{1}^{\prime}(\cdot) / p_{1}(\cdot)}\right)} \\
& \times \sum_{j=-\infty}^{k} 2^{(k-j) w^{+} \alpha^{+}}\left\|v\left(B_{j}\right)^{\alpha_{2}(\cdot) / n} f_{2} \chi_{j}\right\|_{L^{p_{2}(\cdot)}\left(w_{2}\right)}\left\|\chi_{j}\right\|_{L^{p_{2}^{\prime}(\cdot)}\left(w_{2}^{-p_{2}^{\prime}(\cdot) / p_{2}(\cdot)}\right)}\left\|\chi_{k}\right\|_{L^{p(\cdot)}\left(w^{p(\cdot)}\right)} \\
& \lesssim\left\|b_{1}\right\|_{*} \sum_{i=-\infty}^{k} 2^{(k-i)\left(w^{+} \alpha^{+}-n \delta_{12}\right)}\left\|v\left(B_{i}\right)^{\alpha_{1}(\cdot) / n} f_{1} \chi_{i}\right\|_{L^{p_{1}(\cdot)}\left(w_{1}\right)} \\
& \times \sum_{j=-\infty}^{k} 2^{(k-j)\left(w^{+} \alpha^{+}-n \delta_{22}\right)}\left\|v\left(B_{j}\right)^{\alpha_{2}(\cdot) / n} f_{2} \chi_{j}\right\|_{L^{p_{2}(\cdot)}\left(w_{2}\right)} .
\end{aligned}
$$

Since

$$
w=\prod_{i=1}^{2} w_{i}^{p(\cdot) / p_{i}(\cdot)} \quad \text { and } \quad \alpha(\cdot)=\alpha_{1}(\cdot)+\alpha_{2}(\cdot),
$$

by (3.2a), (3.2b), (3.2c), (3.2d) and Hölder's inequality, Lemma 3.4 and (3.3), we have

$$
\begin{aligned}
& \left\|v\left(B_{k}\right)^{\alpha(\cdot) / n} I_{2} \chi_{k}\right\|_{L^{p(\cdot)}(w)} \\
& \lesssim 2^{-2 k n} \sum_{i=-\infty}^{k} 2^{(k-i) w^{+} \alpha^{+}}\left\|v\left(B_{i}\right)^{\alpha_{1}(\cdot) / n} f_{1} \chi_{i}\right\|_{L^{p_{1}(\cdot)}\left(w_{1}\right)}\left\|\left(b_{1}-\left(b_{1}\right)_{B_{i}}\right) \chi_{i}\right\|_{L_{1}^{p^{\prime}(\cdot)}\left(w_{1}^{-p_{1}^{\prime}(\cdot) / p_{1}(\cdot)}\right)} \\
& \quad \times \sum_{j=-\infty}^{k} 2^{(k-j) w^{+} \alpha^{+}}\left\|v\left(B_{j}\right)^{\alpha_{2}(\cdot) / n} f_{2} \chi_{j}\right\|_{L^{p_{2}(\cdot)}\left(w_{2}\right)}\left\|\chi_{j}\right\|_{L^{p_{2}^{\prime}(\cdot)}\left(w_{2}^{-p_{2}^{\prime}(\cdot) / p_{2}(\cdot)}\right)}\left\|\chi_{k}\right\|_{L^{p \cdot(\cdot)}(w)} \\
& \lesssim 2^{-2 k n}\left\|b_{1}\right\|_{*} \sum_{i=-\infty}^{k} 2^{(k-i) w^{+} \alpha^{+}}\left\|v\left(B_{i}\right)^{\alpha_{1}(\cdot) / n} f_{1} \chi_{i}\right\|_{L^{p_{1}(\cdot)}\left(w_{1}\right)}\left\|\chi_{i}\right\|_{L^{p_{1}^{\prime}(\cdot)}\left(w_{1}^{-p_{1}^{\prime}(\cdot) / p_{1}(\cdot)}\right)} \\
& \quad \times \sum_{j=-\infty}^{k} 2^{(k-j) w^{+} \alpha^{+}}\left\|v\left(B_{j}\right)^{\alpha_{2}(\cdot) / n} f_{2} \chi_{j}\right\|_{L^{p_{2}(\cdot)}\left(w_{2}\right)}\left\|\chi_{j}\right\|_{L^{p_{2}^{\prime}(\cdot)}\left(w_{2}^{-p_{2}^{\prime}(\cdot) / p_{2}(\cdot)}\right)}\left\|\chi_{k}\right\|_{L^{p(\cdot)}(w)} \\
& \lesssim\left\|b_{1}\right\|_{*} \sum_{i=-\infty}^{k} 2^{(k-i)\left(w^{+} \alpha^{+}-n \delta_{12}\right)}\left\|v\left(B_{i}\right)^{\alpha_{1}(\cdot) / n} f_{1} \chi_{i}\right\|_{L^{p_{1}(\cdot)}\left(w_{1}\right)}
\end{aligned}
$$




$$
\times \sum_{j=-\infty}^{k} 2^{(k-j)\left(w^{+} \alpha^{+}-n \delta_{22}\right)}\left\|v\left(B_{j}\right)^{\alpha_{2}(\cdot) / n} f_{2} \chi_{j}\right\|_{L^{p_{2}(\cdot)}\left(w_{2}\right)} .
$$

Since

$$
w=\prod_{i=1}^{2} w_{i}^{p(\cdot) / p_{i}(\cdot)} \quad \text { and } \quad \alpha(\cdot)=\alpha_{1}(\cdot)+\alpha_{2}(\cdot)
$$

by (3.2a)-(3.2d) and Hölder's inequality, Lemma 3.4 and (3.3), we have

$$
\begin{aligned}
& \left\|v\left(B_{k}\right)^{\alpha(\cdot) / n} I_{3} \chi_{k}\right\|_{L^{p(\cdot)}(w)} \\
& \lesssim 2^{-2 k n} \sum_{i=-\infty}^{k}\left|\left(b_{1}\right)_{B_{k}}-\left(b_{1}\right)_{B_{i}}\right| 2^{(k-i) w^{+} \alpha^{+}} \\
& \quad \times\left\|v\left(B_{i}\right)^{\alpha_{1}(\cdot) / n} f_{1} \chi_{i}\right\|_{L^{p_{1}(\cdot)}\left(w_{1}\right)}\left\|\chi_{i}\right\|_{L^{p_{1}^{\prime}(\cdot)}\left(w_{1}^{-p_{1}^{\prime}(\cdot) / p_{1}(\cdot)}\right)} \\
& \quad \times \sum_{j=-\infty}^{k} 2^{(k-j) w^{+} \alpha^{+}}\left\|v\left(B_{j}\right)^{\alpha_{2}(\cdot) / n} f_{2} \chi_{j}\right\|_{L^{p_{2}(\cdot)}\left(w_{2}\right)}\left\|\chi_{j}\right\|_{L^{p_{2}^{\prime}(\cdot)}\left(w_{2}{ }^{-p_{2}^{\prime}(\cdot) / p_{2}(\cdot)}\right)}\left\|\chi_{k}\right\|_{L^{p(\cdot)}(w)} \\
& \lesssim 2^{-2 k n}\left\|b_{1}\right\|_{*} \sum_{i=-\infty}^{k}(k-i) 2^{(k-i) w^{+} \alpha^{+}} \\
& \quad \times\left\|v\left(B_{i}\right)^{\alpha_{1}(\cdot) / n} f_{1} \chi_{i}\right\|_{L^{p_{1}(\cdot)}\left(w_{1}\right)}\left\|\chi_{i}\right\|_{L^{p_{1}^{\prime}(\cdot)}\left(w_{1}^{-p_{1}^{\prime}(\cdot) / p_{1}(\cdot)}\right)} \\
& \quad \times \sum_{j=-\infty}^{k} 2^{(k-j) w^{+} \alpha^{+}}\left\|v\left(B_{j}\right)^{\alpha_{2}(\cdot) / n} f_{2} \chi_{j}\right\|_{L^{p_{2}(\cdot)}\left(w_{2}\right)}\left\|\chi_{j}\right\|_{L_{2}^{p_{2}^{\prime}(\cdot)}\left(w_{2}^{-p_{2}^{\prime}(\cdot) / p_{2}(\cdot)}\right)}\left\|\chi_{k}\right\|_{L^{p(\cdot)}(w)} \\
& \lesssim\left\|b_{1}\right\|_{*} \sum_{i=-\infty}^{k}(k-i) 2^{(k-i)\left(w^{+} \alpha^{+}-n \delta_{12}\right)}\left\|v\left(B_{i}\right)^{\alpha_{1}(\cdot) / n} f_{1} \chi_{i}\right\|_{L^{p_{1}(\cdot)}\left(w_{1}\right)} \\
& \quad \times \sum_{j=-\infty}^{k} 2^{(k-j)\left(w^{+} \alpha^{+}-n \delta_{22}\right)}\left\|v\left(B_{j}\right)^{\alpha_{2}(\cdot) / n} f_{2} \chi_{j}\right\|_{L^{p_{2}(\cdot)}\left(w_{2}\right)} \cdot
\end{aligned}
$$

Therefore, we have

$$
\begin{aligned}
& \left\|v\left(B_{k}\right)^{\alpha(\cdot) / n} H_{b_{1}}^{1}\left(f_{1}, f_{2}\right) \chi_{k}\right\|_{L^{p(\cdot)}(w)} \\
& \lesssim\left\|b_{1}\right\|_{*} \sum_{i=-\infty}^{k}(k-i) 2^{(k-i)\left(w^{+} \alpha^{+}-n \delta_{12}\right)}\left\|v\left(B_{i}\right)^{\alpha_{1}(\cdot) / n} f_{1} \chi_{i}\right\|_{L^{p_{1}(\cdot)}\left(w_{1}\right)} \\
& \quad \times \sum_{j=-\infty}^{k} 2^{(k-j)\left(w^{+} \alpha^{+}-n \delta_{22}\right)}\left\|v\left(B_{j}\right)^{\alpha_{2}(\cdot) / n} f_{2} \chi_{j}\right\|_{L^{p_{2}(\cdot)}\left(w_{2}\right)} .
\end{aligned}
$$


By Lemmas 2.1 and 3.2, we have

$$
\begin{aligned}
\left\|H_{b_{1}}^{1}\left(f_{1}, f_{2}\right)\right\|_{\dot{K}_{p(\cdot), \lambda}^{\alpha(\cdot),(\cdot)}(v, w)} \approx & \left(\sum_{k=-\infty}^{-1}\left\|v\left(B_{k}\right)^{\alpha(\cdot) / n} H_{b_{1}}^{1}\left(f_{1}, f_{2}\right) \chi_{k}\right\|_{L^{p(\cdot)}(w)}^{q(0)}\right)^{\frac{1}{q(0)}} \\
& +\left(\sum_{k=0}^{\infty}\left\|v\left(B_{k}\right)^{\alpha(\cdot) / n} H_{b_{1}}^{1}\left(f_{1}, f_{2}\right) \chi_{k}\right\|_{L^{p(\cdot)}(w)}^{q_{\infty}}\right)^{\frac{1}{q_{\infty}}} \\
= & : E+F .
\end{aligned}
$$

Estimate $E$. Since $1 / q(0)=1 / q_{1}(0)+1 / q_{2}(0), \alpha(\cdot)=\alpha_{1}(\cdot)+\alpha_{2}(\cdot)$, by (3.4) and Hölder's inequality, we have

$$
\begin{aligned}
E= & \left(\sum_{k=-\infty}^{-1}\left\|v\left(B_{k}\right)^{\alpha(\cdot) / n} H_{b_{1}}^{1}\left(f_{1}, f_{2}\right) \chi_{k}\right\|_{L^{p(\cdot)}(w)}^{q(0)}\right)^{\frac{1}{q(0)}} \\
\lesssim\left\|b_{1}\right\|_{*} & \left\{\sum _ { k = - \infty } ^ { - 1 } \left(\sum_{i=-\infty}^{k}(k-i) 2^{(k-i)\left(w^{+} \alpha^{+}-n \delta_{12}\right)}\left\|v\left(B_{i}\right)^{\alpha_{1}(\cdot) / n} f_{1} \chi_{i}\right\|_{L^{p_{1}(\cdot)}\left(w_{1}\right)}\right.\right. \\
& \left.\left.\times \sum_{j=-\infty}^{k} 2^{(k-j)\left(w^{+} \alpha^{+}-n \delta_{22}\right)}\left\|v\left(B_{j}\right)^{\alpha_{2}(\cdot) / n} f_{2} \chi_{j}\right\|_{L^{p_{2}(\cdot)}\left(w_{2}\right)}\right)^{q(0)}\right\}^{\frac{1}{q(0)}} \\
\lesssim\left\|b_{1}\right\|_{*} & \left\{\sum_{k=-\infty}^{-1}\left(\sum_{i=-\infty}^{k}\left\|v\left(B_{i}\right)^{\alpha_{1}(\cdot) / n} f_{1} \chi_{i}\right\|_{L^{p_{1}(\cdot)}\left(w_{1}\right)}(k-i) 2^{(k-i)\left(w^{+} \alpha^{+}-n \delta_{12}\right)}\right)^{q_{1}(0)}\right\}^{\frac{1}{q_{1}(0)}} \\
& \times\left\{\sum_{k=-\infty}^{-1}\left(\sum_{j=-\infty}^{k}\left\|v\left(B_{j}\right)^{\alpha_{2}(\cdot) / n} f_{2} \chi_{j}\right\|_{L^{p_{2}(\cdot)}\left(w_{2}\right)} 2^{(k-j)\left(w^{+} \alpha^{+}-n \delta_{22}\right)}\right)^{q_{2}(0)}\right\}^{\frac{1}{q_{2}(0)}} \\
=: & \left\|b_{1}\right\|_{*} E_{1} \times E_{2},
\end{aligned}
$$

where

$$
\begin{aligned}
& E_{1}:=\left\{\sum_{k=-\infty}^{-1}\left(\sum_{i=-\infty}^{k}\left\|v\left(B_{i}\right)^{\alpha_{1}(\cdot) / n} f_{1} \chi_{i}\right\|_{L^{p_{1}(\cdot)}\left(w_{1}\right)}(k-i) 2^{(k-i)\left(w^{+} \alpha^{+}-n \delta_{12}\right)}\right)^{q_{1}(0)}\right\}^{\frac{1}{q_{1}(0)}}, \\
& E_{2}:=\left\{\sum_{k=-\infty}^{-1}\left(\sum_{j=-\infty}^{k}\left\|v\left(B_{j}\right)^{\alpha_{2}(\cdot) / n} f_{2} \chi_{j}\right\|_{L^{p_{2}(\cdot)}\left(w_{2}\right)} 2^{(k-j)\left(w^{+} \alpha^{+}-n \delta_{22}\right)}\right)^{q_{2}(0)}\right\}^{\frac{1}{q_{2}(0)}} .
\end{aligned}
$$

Estimate $E_{1}$. Since $w^{+} \alpha^{+}-n \delta_{12}<0$, we write

$$
(k-i) 2^{-|k-i|\left(n \delta_{12}-w^{+} \alpha^{+}\right)} \lesssim 2^{-|k-i| \varepsilon_{1}}
$$

for some $\varepsilon_{1} \in\left(0, n \delta_{12}-w^{+} \alpha^{+}\right)$, in Lemma 3.7 replacing $\delta$ by $\varepsilon_{1}$, we obtain that

$$
E_{1} \lesssim\left(\sum_{i=-\infty}^{-1}\left\|\left[v\left(B_{i}\right)\right]^{\alpha_{1}(\cdot) / n} f_{1} \chi_{i}\right\|_{L^{p_{1}(\cdot)}\left(w_{1}\right)}^{q_{1}(0)}\right)^{\frac{1}{q_{1}(0)}} \lesssim\left\|f_{1}\right\|_{\dot{K}_{p_{1}(\cdot), \lambda_{1}}^{\alpha_{1}(\cdot), q_{1}(\cdot)}\left(v, w_{1}\right)} .
$$


Estimate $E_{2}$. Since $w^{+} \alpha^{+}-n \delta_{22}<0$, in Lemma 3.7 replacing $\delta$ by $n \delta_{22}-w^{+} \alpha^{+}$, we obtain that

$$
E_{2} \lesssim\left(\sum_{j=-\infty}^{-1}\left\|v\left(B_{j}\right)^{\alpha_{2}(\cdot) / n} f_{2} \chi_{j}\right\|_{L^{p_{2}(\cdot)}\left(w_{2}\right)}^{q_{2}(0)}\right)^{\frac{1}{q_{2}(0)}} \lesssim\left\|f_{2}\right\|_{\dot{K}_{p_{2}(\cdot), \lambda_{2}}^{\alpha_{2}(\cdot), q_{2}(\cdot)}\left(v, w_{2}\right)} .
$$

Thus, we get

$$
E \lesssim\left\|b_{1}\right\|_{*}\left\|f_{1}\right\|_{\dot{K}_{p_{1}(\cdot), \lambda_{1}}^{\alpha_{1}(\cdot), q_{1}(\cdot)}\left(v, w_{1}\right)}\left\|f_{2}\right\|_{\dot{K}_{p_{2}(\cdot), \lambda_{2}}^{\alpha_{2}(\cdot), q_{2}(\cdot)}\left(v, w_{2}\right)} .
$$

Estimate $F$. Since $1 / q_{\infty}=1 / q_{1 \infty}+1 / q_{2 \infty}, \alpha(\cdot)=\alpha_{1}(\cdot)+\alpha_{2}(\cdot)$, by (3.4) and Hölder's inequality, we have

$$
\begin{aligned}
F=\left(\sum_{k=0}^{\infty}\left\|v\left(B_{k}\right)^{\alpha(\cdot) / n} H_{b_{1}}^{1}\left(f_{1}, f_{2}\right) \chi_{k}\right\|_{L^{p(\cdot)}(w)}^{q_{\infty}}\right)^{\frac{1}{q_{\infty}}} \\
\lesssim\left\|b_{1}\right\|_{*}\left\{\sum _ { k = 0 } ^ { \infty } \left(\sum_{i=-\infty}^{k}\left\|v\left(B_{i}\right)^{\alpha_{1}(\cdot) / n} f_{1} \chi_{i}\right\|_{L^{p_{1}(\cdot)\left(w_{1}\right)}}(k-i) 2^{(k-i)\left(w^{+} \alpha^{+}-n \delta_{12}\right)}\right.\right. \\
\left.\left.\times \sum_{j=-\infty}^{k}\left\|v\left(B_{j}\right)^{\alpha_{2}(\cdot) / n} f_{2} \chi_{j}\right\|_{L^{p_{2}(\cdot)}\left(w_{2}\right)} 2^{(k-j)\left(w^{+} \alpha^{+}-n \delta_{22}\right)}\right)^{q_{\infty}}\right\}^{\frac{1}{q_{\infty}}} \\
\lesssim\left\|b_{1}\right\|_{*}\left\{\sum_{k=0}^{\infty}\left(\sum_{i=-\infty}^{k}\left\|v\left(B_{i}\right)^{\alpha_{1}(\cdot) / n} f_{1} \chi_{i}\right\|_{L^{p_{1}(\cdot)\left(w_{1}\right)}}(k-i) 2^{(k-i)\left(w^{+} \alpha^{+}-n \delta_{12}\right)}\right)^{q_{1 \infty}}\right\}^{\frac{1}{q_{1 \infty}}} \\
\times\left\{\sum_{k=0}^{\infty}\left(\sum_{j=-\infty}^{k}\left\|v\left(B_{j}\right)^{\alpha_{2}(\cdot) / n} f_{2} \chi_{j}\right\|_{L^{p_{2}(\cdot)}\left(w_{2}\right)} 2^{(k-j)\left(w^{+} \alpha^{+}-n \delta_{22}\right)}\right)^{q_{2 \infty}}\right\}^{\frac{1}{q_{2 \infty}}} \\
=:\left\|b_{1}\right\|_{*} F_{1} \times F_{2},
\end{aligned}
$$

where

$$
\begin{aligned}
& F_{1}:=\left\{\sum_{k=0}^{\infty}\left(\sum_{i=-\infty}^{k}\left\|v\left(B_{i}\right)^{\alpha_{1}(\cdot) / n} f_{1} \chi_{i}\right\|_{L^{p_{1}(\cdot)}\left(w_{1}\right)}(k-i) 2^{(k-i)\left(w^{+} \alpha^{+}-n \delta_{12}\right)}\right)^{q_{1 \infty}}\right\}^{\frac{1}{q_{1 \infty}}}, \\
& F_{2}:=\left\{\sum_{k=0}^{\infty}\left(\sum_{j=-\infty}^{k}\left\|v\left(B_{j}\right)^{\alpha_{2}(\cdot) / n} f_{2} \chi_{j}\right\|_{L^{p_{2}(\cdot)}\left(w_{2}\right)} 2^{(k-j)\left(w^{+} \alpha^{+}-n \delta_{22}\right)}\right)^{q_{2 \infty}}\right\}^{\frac{1}{q_{2 \infty}}} .
\end{aligned}
$$

Estimate $F_{1}$. we obtain that

$$
\begin{aligned}
F_{1} \lesssim\left\{\sum _ { k = 0 } ^ { \infty } \left(\sum_{i=-\infty}^{-1}\left\|v\left(B_{i}\right)^{\alpha_{1}(\cdot) / n} f_{1} \chi_{i}\right\|_{L^{p_{1}(\cdot)}\left(w_{1}\right)}(k-i) 2^{(k-i)\left(w^{+} \alpha^{+}-n \delta_{12}\right)}\right.\right. \\
\left.\left.+\sum_{i=0}^{k}(k-i)\left\|v\left(B_{i}\right)^{\alpha_{1}(\cdot) / n} f_{1} \chi_{i}\right\|_{L^{p_{1}(\cdot)}\left(w_{1}\right)} 2^{(k-i)\left(w^{+} \alpha^{+}-n \delta_{12}\right)}\right)^{q_{1 \infty}}\right\}^{\frac{1}{q_{1 \infty}}}
\end{aligned}
$$




$$
\begin{aligned}
& \lesssim\left\{\sum_{k=0}^{\infty}\left(\sum_{i=-\infty}^{-1}\left\|v\left(B_{i}\right)^{\alpha_{1}(\cdot) / n} f_{1} \chi_{i}\right\|_{L^{p_{1}(\cdot)}\left(w_{1}\right)}(k-i) 2^{(k-i)\left(w^{+} \alpha^{+}-n \delta_{12}\right)}\right)^{q_{1 \infty}}\right\}^{\frac{1}{q_{1 \infty}}} \\
& \quad+\left\{\sum_{k=0}^{\infty}\left(\sum_{i=0}^{k}\left\|v\left(B_{i}\right)^{\alpha_{1}(\cdot) / n} f_{1} \chi_{i}\right\|_{L^{p_{1}(\cdot)}\left(w_{1}\right)}(k-i) 2^{(k-i)\left(w^{+} \alpha^{+}-n \delta_{12}\right)}\right)^{q_{1 \infty}}\right\}^{\frac{1}{q_{1 \infty}}} \\
& =: F_{1,1}+F_{1,2} .
\end{aligned}
$$

Estimate $F_{1,1}$. Since $w^{+} \alpha^{+}-n \delta_{12}<0$, we obtain that

$$
\begin{aligned}
F_{1,1} & =\left\{\sum_{k=0}^{\infty}\left(\sum_{i=-\infty}^{-1}\left\|v\left(B_{i}\right)^{\alpha_{1}(\cdot) / n} f_{1} \chi_{i}\right\|_{L^{p_{1}(\cdot)}\left(w_{1}\right)}(k-i) 2^{(k-i)\left(w^{+} \alpha^{+}-n \delta_{12}\right)}\right)^{q_{1 \infty}}\right\}^{\frac{1}{q_{1 \infty}}} \\
& \lesssim\left\|f_{1}\right\|_{\dot{K}_{p_{1}(\cdot)}^{\alpha_{1}(\cdot), q_{1}(\cdot)}\left(v, w_{1}\right)}\left\{\sum_{k=0}^{\infty}\left(\sum_{i=-\infty}^{-1}(k-i) 2^{(k-i)\left(w^{+} \alpha^{+}-n \delta_{12}\right)}\right)^{q_{1 \infty}}\right\}^{\frac{1}{q_{1 \infty}}} \\
& \lesssim\left\|f_{1}\right\|_{\dot{K}_{p_{1}}^{\alpha_{1}(\cdot), q_{1}(\cdot)}\left(v, w_{1}\right)}\left\{\sum_{k=0}^{\infty} k^{q_{1 \infty}} 2^{k q_{1 \infty}\left(w^{+} \alpha^{+}-n \delta_{12}\right)}\left(\sum_{i=-\infty}^{-1}(-i) 2^{(-i)\left(w^{+} \alpha^{+}-n \delta_{12}\right)}\right)^{q_{1 \infty}}\right\}^{\frac{1}{q_{1 \infty}}} \\
& \lesssim\left\|f_{1}\right\|_{\dot{K}_{p_{1}(\cdot)}^{\alpha_{1}(\cdot), q_{1}(\cdot)}\left(v, w_{1}\right)} .
\end{aligned}
$$

Estimate $F_{1,2}$. Since $w^{+} \alpha^{+}-n \delta_{12}<0$, we write

$$
(k-i) 2^{-|k-i|\left(n \delta_{12}-w^{+} \alpha^{+}\right)} \lesssim 2^{-|k-i| \eta_{1}}
$$

for some $\eta_{1} \in\left(0, n \delta_{12}-w^{+} \alpha^{+}\right)$, then in Lemma 3.7 replacing $\delta$ by $\eta_{1}$, we have

$$
\begin{aligned}
F_{1,2} & =\left\{\sum_{k=0}^{\infty}\left(\sum_{i=0}^{k}\left\|v\left(B_{i}\right)^{\alpha_{1}(\cdot) / n} f_{1} \chi_{i}\right\|_{L^{p_{1}(\cdot)}\left(w_{1}\right)}(k-i) 2^{(k-i)\left(w^{+} \alpha^{+}-n \delta_{12}\right)}\right)^{q_{1 \infty}}\right\}^{\frac{1}{q_{1 \infty}}} \\
& \lesssim\left(\sum_{i=0}^{\infty}\left\|v\left(B_{i}\right)^{\alpha_{1}(\cdot) / n} f_{1} \chi_{i}\right\|_{L^{p_{1}(\cdot)}\left(w_{1}\right)}^{q_{1 \infty}}\right)^{\frac{1}{q_{1 \infty}}} \\
& \lesssim\left\|f_{1}\right\|_{M \dot{K}_{p_{1}(\cdot), \lambda_{1}}^{\alpha_{1}(\cdot), q_{1}(\cdot)}\left(w_{1}\right)} \cdot
\end{aligned}
$$

For $F_{2}$, we obtain that

$$
\begin{aligned}
F_{2} \lesssim\left\{\sum _ { k = 0 } ^ { \infty } \left(\sum_{j=-\infty}^{-1}\left\|v\left(B_{j}\right)^{\alpha_{2}(\cdot) / n} f_{2} \chi_{j}\right\|_{L^{p_{2}(\cdot)}\left(w_{2}\right)} 2^{(k-j)\left(w^{+} \alpha^{+}-n \delta_{22}\right)}\right.\right. \\
\left.\left.+\sum_{j=0}^{k}\left\|v\left(B_{j}\right)^{\alpha_{2}(\cdot) / n} f_{2} \chi_{j}\right\|_{L^{p_{2}(\cdot)}\left(w_{2}\right)} 2^{(k-j)\left(w^{+} \alpha^{+}-n \delta_{22}\right)}\right)^{q_{2 \infty}}\right\}^{\frac{1}{q_{2 \infty}}}
\end{aligned}
$$




$$
\begin{aligned}
& \lesssim\left\{\sum_{k=0}^{\infty}\left(\sum_{j=-\infty}^{-1}\left\|v\left(B_{j}\right)^{\alpha_{2}(\cdot) / n} f_{2} \chi_{j}\right\|_{L^{p_{2}(\cdot)}\left(w_{2}\right)} 2^{(k-j)\left(w^{+} \alpha^{+}-n \delta_{22}\right)}\right)^{q_{2 \infty}}\right\}^{\frac{1}{q_{2 \infty}}} \\
& \quad+\left\{\sum_{k=0}^{\infty}\left(\sum_{j=0}^{k}\left\|v\left(B_{j}\right)^{\alpha_{2}(\cdot) / n} f_{2} \chi_{j}\right\|_{L^{p_{2}(\cdot)}\left(w_{2}\right)} 2^{(k-j)\left(w^{+} \alpha^{+}-n \delta_{22}\right)}\right)^{q_{2 \infty}}\right\}^{\frac{1}{q_{2 \infty}}} \\
& =: F_{2,1}+F_{2,2} .
\end{aligned}
$$

Since $w^{+} \alpha^{+}-n \delta_{22}<0$, we have

$$
\begin{aligned}
F_{2,1} & =\left\{\sum_{k=0}^{\infty}\left(\sum_{j=-\infty}^{-1}\left\|v\left(B_{j}\right)^{\alpha_{2}(\cdot) / n} f_{2} \chi_{j}\right\|_{L^{p_{2}(\cdot)}\left(w_{2}\right)} 2^{(k-j)\left(w^{+} \alpha^{+}-n \delta_{22}\right)}\right)^{q_{2 \infty}}\right\}^{\frac{1}{q_{2 \infty}}} \\
& \lesssim\left\|f_{2}\right\|_{\dot{K}_{p_{2}(\cdot)}^{\alpha_{2}(\cdot), q_{2}(\cdot)}\left(v, w_{2}\right)}\left\{\sum_{k=0}^{\infty}\left(\sum_{j=-\infty}^{-1} 2^{(k-j)\left(w^{+} \alpha^{+}-n \delta_{22}\right)}\right)^{q_{2 \infty}}\right\}^{\frac{1}{q_{2 \infty}}} \\
& \lesssim\left\|f_{2}\right\|_{\dot{K}_{p_{2}(\cdot)}^{\alpha_{2}(\cdot), q_{2}(\cdot)}\left(v, w_{2}\right)} \cdot
\end{aligned}
$$

Since $w^{+} \alpha^{+}-n \delta_{22}<0$, where wrote

$$
2^{-|k-j|\left(n \delta_{22}-w^{+} \alpha^{+}\right)} \lesssim 2^{-|k-j| \eta_{2}}
$$

for some $\eta_{2} \in\left(0, n \delta_{22}-w^{+} \alpha^{+}\right)$, then in Lemma 3.7 replacing $\delta$ by $\eta_{2}$, we have

$$
\begin{aligned}
F_{2,2} & =\left\{\sum_{k=0}^{\infty}\left(\sum_{j=0}^{k}\left\|\left[v\left(B_{j}\right)\right]^{\alpha_{2}(\cdot) / n} f_{2} \chi_{j}\right\|_{L^{p_{2}(\cdot)}\left(w_{2}\right)} 2^{(k-j)\left(w^{+} \alpha^{+}-n \delta_{22}\right)}\right)^{q_{2 \infty}}\right\}^{\frac{1}{q_{2 \infty}}} \\
& \lesssim\left(\sum_{j=0}^{\infty}\left\|v\left(B_{j}\right)^{\alpha_{2}(\cdot) / n} f_{2} \chi_{i}\right\|_{L^{p_{2}(\cdot)}\left(w_{2}\right)}^{q_{2 \infty}}\right)^{\frac{1}{q_{2 \infty}}} \\
& \lesssim\left\|f_{2}\right\|_{\dot{K}_{p_{2}(\cdot)}^{\alpha_{2}\left(\cdot, q_{2}(\cdot)\right.}\left(v, w_{2}\right)} .
\end{aligned}
$$

Thus, we get

$$
H \lesssim\left\|b_{1}\right\|_{*}\left\|f_{1}\right\|_{\dot{K}_{p_{1}(\cdot)}^{\alpha_{1}(\cdot), q_{1}(\cdot)}\left(v, w_{1}\right)}\left\|f_{2}\right\|_{\dot{K}_{p_{2}(\cdot)}^{\alpha_{2}(\cdot), q_{2}(\cdot)}\left(v, w_{2}\right)} .
$$

Combining $E$ and $F$, we obtain that

$$
\left\|H_{b_{1}}^{1}\left(f_{1}, f_{2}\right)\right\|_{\dot{K}_{p(\cdot)}^{\alpha(\cdot), q(\cdot)}(v, w)} \lesssim\left\|b_{1}\right\|_{*}\left\|f_{1}\right\|_{\dot{K}_{p_{1}(\cdot)}^{\alpha_{1}(\cdot), q_{1}(\cdot)}\left(v, w_{1}\right)}\left\|f_{2}\right\|_{\dot{K}_{p_{2}(\cdot)}^{\alpha_{2}(\cdot), q_{2}(\cdot)}\left(v, w_{2}\right)} .
$$

Similarly, we have

$$
\left\|H_{b_{2}}^{1}\left(f_{1}, f_{2}\right)\right\|_{\dot{K}_{p(\cdot)}^{\alpha(\cdot), q(\cdot)}(v, w)} \lesssim\left\|b_{2}\right\|_{*}\left\|f_{1}\right\|_{\dot{K}_{p_{1}(\cdot)}^{\alpha_{1}(\cdot), q_{1}(\cdot)}\left(v, w_{1}\right)}\left\|f_{2}\right\|_{\dot{K}_{p_{2}(\cdot)}^{\alpha_{2}(\cdot), q_{2}(\cdot)}\left(v, w_{2}\right)} .
$$

Therefore, the proof of Theorem 2.1 is completed. 


\section{Acknowledgements}

The second author would like to express thanks to Professor Shanzhen Lu for his supervision and constant supports. The second author is partially supported by the National Natural Science Foundation of China (Grant No. 11761026) and Guangxi Natural Science Foundation (Grant No. 2020GXNSFAA159085).

\section{References}

[1] A. Almeida and D. Drihem, Maximal, potential and singular type operators on Herz spaces with variable exponents, J. Math. Anal. Appl., 394 (2012), 781-795.

[2] A. Nekvinda, Hardy-Littlewood maximal operator on $L^{p(x)}\left(\mathbb{R}^{n}\right)$, Math. Inequal. Appl., 7 (2004), 255-266.

[3] A. Torchinsky, Real-Variable Methods in Harmonic Analysis, Pure and Applied Mathematics, vol. 123, Academic Press Inc., Orlando, 1986.

[4] A. W. Huang, and J. S. Xu, Multilinear singular integral and commutators in variable exponent Lebesgue space, Appl. Math. J. Chinese Univ. Ser. B, 25 (2010), 69-77.

[5] B. Muckenhoupt, Weighted norm inequalities for the Hardy maximal function, Tran. Amer. Math. Soci., 165 (1972), 207-226.

[6] D. Cruz-Uribe, A. Fiorenza, J. M. Martell and C. Pérez, The boundedness of classical operators on variable $L^{p}$ spaces, Ann. Acad. Sci. Fenn. Math., 31 (2006), 239-264.

[7] F. Y. Zhao and S. Z. Lu, The best bound for $n$-dimensional fractional Hardy operators, Math. Inequal. Appl., 18 (2015), 233-240.

[8] F. Y. Zhao, Z . W. Fu and S. Z. Lu, $M_{p}$ weights for bilinear Hardy operators on $\mathbb{R}^{n}$, Collect. Math., 65 (2014), 87-102.

[9] F. Y. Zhao, Z. W. Fu and S. Z. Lu, Endpoint estimates for n-dimensional Hardy operators and their commutators, Sci. China Math., 55 (2012), 1977-1990.

[10] G. H. Hardy, Note on a theorem of Hilbert, Math Z., 6 (1920), 314-317.

[11] J. Duoandikoetxea, Fourier analysis, in: Graduate Studies in Mathematics, vol. 29, American Mathematical Society, Providence, RI (2001).

[12] J. García-Cuerva and J. L. de Rubio Francia, Weighted Norm Inequalities and Related Topics, North-Holland Mathematics Studies, vol. 116, North-Holland, Amsterdam, 1985.

[13] K.-P. Ho, Atomic decomposition of Hardy spaces and characterization of BMO via Banach function spaces, Anal. Math., 38 (2012), 173-185.

[14] L. Diening, Maximal function on generalized Lebesgue spaces $L^{p(\cdot)}$, Math. Inequal. Appl., 7 (2004), 245-253.

[15] L. Diening, P. Harjulehto, P. Hästö and M. Ruzicka, Lebesgue and Sobolev Spaces with Variable Exponents, Springer, Heidelberg, 2011.

[16] L. Diening, P. Harjulehto, P. Hästö, Y. Mizuta and T. Shimomura, Maximal functions in variable exponent spaces: limiting cases of the exponent, Ann. Acad. Sci. Fenn. Math., 34 (2009), 503-522.

[17] L. Shu, M. Wang and Q. Meng, Commutators of Hardy type operators on Herz spaces with variable exponents (Chinese), Acta Math. Sin., 58 (2015), $29-40$.

[18] M. Christ and L. Grafakos, Best constants for two nonconvolution inequalities, Proc. Amer. Math. Soc., 123 (1995), 1687-1693. 
[19] M. Izuki, Boundedness of commutators on Herz spaces with variable exponent, Rend. Circ. Mat. Palermo(2), 59 (2010), 199-213.

[20] M. Izuki, Another proof of characterization of BMO via Banach function spaces, Revista del aunión Mat. Argent., 57 (2016), 103-109.

[21] M. Izuki, Remarks on Muckenhoupt weights with variable exponent, J. Anal. Appl., 11 (2013), 27-41.

[22] M. Izuki, Y. Sawano and Y. Tsutsui, Variable Lebesgue norm estimates for BMO functions, II, Anal. Math., 40 (2014), 215-230.

[23] M. Izuki and T. Noi, Two weighted Herz spaces with variable exponents, Bull. Malays. Math. Sci. Soc., 43 (2020), 169-200.

[24] M. Izuki and T. Noi, Boundedness of fractional integrals on weighted Herz spaces with variable exponent, J. Inequal. Appl., 2016 (2016), Article ID 199.

[25] S. Z. Lu, Some recent progress of n-dimensional Hardy operators, Adv. Math. (China), 42 (2013), 737-747.

[26] S. Z. Lu, D. Y. Yan and F. Y. Zhao, Sharp bounds for Hardy type operators on higherdimensional product spaces, J. Inequal. Appl., 2013 (2013), Article ID 148.

[27] S. G. Shi and S. Z. Lu, Characterization of the central Campanato space via the commutator operator of Hardy type, J. Math. Anal. Appl., 429 (2015), 713-732.

[28] W. G. Faris, Week Lebesgue spaces and quantum mechanical binding, Duke Math. J., 43 (1967), 365-373.

[29] X. Yu and S. Z. Lu, Endpoint estimates for generalized commutators of Hardy operators on $H^{1}$ space, J. Funct. Spaces Appl., 2013 (2013), Article ID 410305.

[30] Y. Sawano, Theory of Besov Spaces, Springer, Singapore, 2018.

[31] Z. W. Fu, Z. G. Liu and S. Z. Lu, Commutators of weighted Hardy operators on $\mathbb{R}^{n}$, Proc. Amer. Math. Soc., 137 (2009), 3319-3328.

[32] Z. W. Fu, L. Grafakos, S. Z. Lu and F. Y. Zhao, Sharp bounds for $m$-linear Hardy and Hilbert operators, Houston J. Math., 38 (2012), 225-244.

[33] Z. W. Fu, Q. Y. Wu and S. Z. Lu, Sharp estimates of $p$-adic Hardy and Hardy-LittlewoodPólya operators, Acta Math. Sin. (Engl. Ser.), 29 (2013), 137-150.

[34] Z. W. Fu, S. L. Gong, S. Z. Lu and W. Yuan, Weighted multilinear Hardy operators and commutators, Forum Math., 27 (2015), 2825-2851. 